\title{
Nearly complete graphs decomposable into large induced matchings and their applications
}

Received August 14, 2012 and in revised form January 28, 2013

\begin{abstract}
We describe two constructions of (very) dense graphs which are edge disjoint unions of large induced matchings. The first construction exhibits graphs on $N$ vertices with $\left(\begin{array}{c}N \\ 2\end{array}\right)-o\left(N^{2}\right)$ edges, which can be decomposed into pairwise disjoint induced matchings, each of size $N^{1-o(1)}$. The second construction provides a covering of all edges of the complete graph $K_{N}$ by two graphs, each being the edge disjoint union of at most $N^{2-\delta}$ induced matchings, where $\delta>0.076$. This disproves (in a strong form) a conjecture of Meshulam, substantially improves a result of Birk, Linial and Meshulam on communicating over a shared channel, and (slightly) extends the analysis of Håstad and Wigderson of the graph test of Samorodnitsky and Trevisan for linearity. Additionally, our constructions settle a combinatorial question of Vempala regarding a candidate rounding scheme for the directed Steiner tree problem.
\end{abstract}

\section{Introduction}

\subsection{Background}

Dense graphs consisting of large pairwise edge disjoint induced matchings have found several applications in combinatorics, complexity theory and information theory. Call a graph $G=(V, E)$ an $(r, t)$-Ruzsa-Szemerédi graph $((r, t)$-RS graph, for short $)$ if its set of edges consists of $t$ pairwise disjoint induced matchings, each of size $r$. The total number of edges of such a graph is clearly $r t$. Graphs of this type are useful when both $r$ and $t$ are relatively large as a function of the number of vertices $N$. There are several known interesting constructions, relying on a variety of techniques.

The first surprising construction was given by Ruzsa and Szemerédi [23], who applied a result of Behrend [7] about the existence of dense subsets of $\{1, \ldots, \Theta(N)\}$ containing no 3-term arithmetic progressions to prove that there are $(r, t)$-RS graphs on $N$ vertices with $r=N / e^{O(\sqrt{\log N})}$ and $t=N / 3$. They applied this construction, together with the regularity lemma of Szemerédi [26], to settle an extremal problem of Brown, Erdós and

N. Alon: Sackler School of Mathematics and Blavatnik School of Computer Science, Tel Aviv University, Tel Aviv 69978, Israel, and Institute for Advanced Study, Princeton, NJ, 08540, USA; e-mail:nogaa@tau.ac.il

A. Moitra: Institute for Advanced Study, Princeton, NJ, 08540, USA; e-mail: moitra@ias.edu

B. Sudakov: Department of Mathematics, UCLA, Los Angeles, CA 90095, USA;

e-mail: bsudakov@math.ucla.edu 
Sós [11, 12], showing that the maximum possible number of edges in a 3-uniform hypergraph on $N$ vertices which contains no 6 vertices spanning at least 3 edges is greater than $N^{2-\epsilon}$ and smaller than $\epsilon N^{2}$, for any $\epsilon>0$, provided $N>N_{0}(\epsilon)$. See also [14], [5] for more details about this problem, its extensions, and their connection to $(r, t)$-RS graphs.

Note that the above construction provides graphs with $N$ vertices and $N^{2} / e^{O(\sqrt{\log N})}$ edges, that is, rather dense graphs, but still ones in which the number of edges is $o\left(N^{2}\right)$. These graphs and some appropriate variants have been used by the first author in [1], to show that the problem of testing $H$-freeness in graphs requires a super-polynomial (in $1 / \epsilon$ ) number of queries if and only if $H$ is not bipartite. The proof for one-sided error algorithms is given in [1], and an extension for two-sided algorithms is described in [3]. A similar application of these graphs for testing induced $H$-freeness appears in [4], and yet another very recent application showing that testing graph-perfectness requires a super-polynomial number of queries appears in [2].

The above graphs have also been applied by Håstad and Wigderson [20] to give an improved analysis of the graph test of Samorodnitsky and Trevisan for linearity and for PCP with low amortized complexity [24].

Another construction of $(r, t)$-RS graphs on $N$ vertices, with $r=N / 3-o(N)$ and $t=N^{\Omega(1 / \log \log N)}$, was given by Fischer et al. in [15]. Note that the matchings here are of linear size, but their number is much smaller than in the original construction of Ruzsa and Szemerédi. The construction here is combinatorial, and Fischer et al. use these graphs to establish an $N^{\Omega(1 / \log \log N)}$ lower bound for testing monotonicity in general posets.

Yet another construction was obtained by Birk, Linial and Meshulam [8], and in an improved form by Meshulam [22]. For the application in [8] it is crucial to obtain graphs with positive density. Indeed, the graphs here are $(r, t)$-graphs on $N$ vertices with $r=$ $(\log N)^{\Omega\left(\log \log N /(\log \log \log N)^{2}\right)}$ and $t$ roughly $N^{2} / 24 r$. Thus, their number of edges is about $N^{2} / 24$. The method here relies on a construction of a low degree representation of the OR function, due to Barrington, Beigel and Rudich [6]. The application in [8] is in information theory, the graphs are applied to design an efficient deterministic scheduling scheme for communicating over a shared directional multichannel.

Interestingly, none of these constructions address the question of whether or not an $(r, t)$-RS graph can simultaneously have positive density and yet be an edge disjoint union of polynomially large induced matchings. This range of parameters is important for some applications - especially ones in which there is a tradeoff between the number of missing edges and the number of induced matchings needed to cover the graph. Indeed, Meshulam [22] conjectured that there were no such graphs. We are able to disprove this conjecture in the strongest possible sense: The density of our construction is $1-o(1)$ and yet $r$ is nearly linear in $N$. We also give a number of applications of our constructions.

\subsection{Our results}

We construct $(r, t)$-RS graphs on $N$ vertices with $r t=(1-o(1))\left(\begin{array}{c}N \\ 2\end{array}\right)$, and $r=N^{1-o(1)}$. Thus, not only can we have graphs with positive edge density which are edge disjoint union of induced matchings of size $N^{\Omega(1)}$, but in fact we can have edge density $1-o(1)$, where the size of each matching is $N^{1-o(1)}$. We also describe another construction of a 
partition of the complete graph $K_{N}$ into two subgraphs, each being a union of at most $N^{2-\delta}$ induced matchings, where $\delta>0.076$. The main difference between the new constructions presented here and the previous ones mentioned above is that the graphs constructed here are of density $1-o(1)$, that is, almost all edges of the complete graph $K_{N}$ are covered, and yet all these edges can be partitioned into large pairwise disjoint induced matchings. This surprising property turns out to be useful in various applications.

Our first construction is geometric, and is inspired by the recent work of Fox and Loh [17] on dense graphs in which every edge is contained in at least one triangle and yet no edge is contained in too many triangles. The construction follows the basic approach of Fox and Loh (slightly modified according to the remark of the first author, mentioned at the end of [17]) with different parameters. An additional (simple) argument is required in decomposing sparse graphs into not too many induced matchings. Our second construction applies some basic tools from coding theory. Also we make use of the regularity lemma and some combinatorial and entropy based techniques to prove lower bounds for these questions.

It is worth noting that a general result of Frankl and Füredi [18] implies that for any fixed $r$, there are $(r, t)$-RS graphs $G$ on $N$ vertices with $r t=(1-o(1))\left(\begin{array}{c}N \\ 2\end{array}\right)$. This is proved by choosing the non-edges of $G$ randomly and by applying the nibble technique to obtain the existence of the desired matchings. This method, however, yields the induced matchings of constant size, whereas we are interested, crucially, in large matchings. The techniques of [18] cannot provide induced matchings of size exceeding $\Theta(\log N)$.

We apply our results to significantly improve the application in [8]. As mentioned earlier, Birk, Linial and Meshulam construct $(r, t)$-graphs on $N$ vertices with $r=$ $(\log N)^{\Omega\left(\log \log N /(\log \log \log N)^{2}\right)}$ and $r t$ roughly $N^{2} / 24$. The authors then use these graphs to design a communication protocol over a shared directional multi-channel-it is critical for this application that these graphs have positive density. The communication protocol based on these graphs achieves a round complexity of $O\left(N^{2} / r\right)$ and this is a slightly better than poly logarithmic improvement over the naive protocol for bus-based architectures.

We can use our construction to achieve a round complexity of $O\left(N^{2-\delta}\right)$ over a shared directional multi-channel. This is the first such protocol that is a polynomial improvement over the naive protocol. We can accomplish this using just two receivers per station (this corresponds to a partition of the edges of a complete bipartite graph into two graphs that can be decomposed into large induced matchings). In case we are allowed $C=C(\epsilon)$ receivers per station, we can achieve a round complexity that is $O\left(N^{1+\epsilon}\right)$ for any $\epsilon>0$. Hence, previous protocols required nearly a quadratic number of rounds, and our protocols require only a nearly linear number of rounds.

Our constructions also disprove the recent conjecture of Meshulam. Moreover, we can achieve a density approaching 1 while simultaneously being able to decompose the graph into at most a nearly linear number of induced matchings.

Besides their applications to the problems of [8] and [22], our constructions can be plugged in the result of [20], extending it to a new range of the parameters that may be of interest. Lastly, we also answer a question of Vempala [27], showing that a certain rounding scheme for the directed Steiner tree problem is not effective. 
The rest of this paper is organized as follows. In the next section we describe the two new constructions. Lower bounds showing that these are not far from being tight are given in Section 3. In Section 4 we describe applications of these graphs. The final Section 5 contains some concluding remarks and open problems.

\section{Constructions}

\subsection{A geometric construction}

Here we construct nearly complete graphs that can be covered by an almost linear number of induced matchings. These graphs will be based on a geometric construction inspired by a recent construction of Fox and Loh [17].

We first describe a graph $G=(V, E)$ and then prove that it can be slightly modified to yield a nearly complete $(r, t)$-RS graph. Set $V=[C]^{n}$ for some constant $C$ to be chosen later. Let $N=C^{n}$ be the number of vertices. Each vertex $x \in V$ will be interpreted as an integer vector in $n$ dimensions with coordinates in $[C]=\{1, \ldots, C\}$, where for technical reasons it is convenient to assume that $n$ is even. Let $\mu=E_{x, y}\left[\|x-y\|_{2}^{2}\right]$ where $x$ and $y$ are sampled uniformly at random from $V$. It is easy to show that $\mu=\frac{1}{6} n\left(c^{2}-1\right)$, but we will not need this exact value.

Next, we describe the set $E$ of edges. A pair of vertices $x$ and $y$ are adjacent if and only if

$$
\left|\|x-y\|_{2}^{2}-\mu\right| \leq n
$$

This condition implies that the number of missing edges is small, by a standard application of Hoeffding's inequality:

Claim 2.1. $\left(\begin{array}{c}N \\ 2\end{array}\right)-|E| \leq\left(\begin{array}{c}N \\ 2\end{array}\right) 2 e^{-n / 2 C^{4}}$.

Proof. The quantity $\|x-y\|_{2}^{2}=\sum_{i=1}^{n}\left(x_{i}-y_{i}\right)^{2}$ is the sum of independent random variables (when $x$ and $y$ are chosen uniformly at random from $V$ ). Each variable is bounded in the range $\left[0, C^{2}\right]$ and hence we can apply Hoeffding's inequality to obtain

$$
\operatorname{Pr}\left[\left|\|x-y\|_{2}^{2}-\mu\right|>n\right] \leq 2 e^{-n / 2 C^{4}},
$$

which implies the claim.

As a first step, we will cover all the edges of $G$ by a linear number of induced subgraphs of small (but super-constant) maximum degree. We will then use this covering to obtain a covering via an almost linear number of induced matchings. Next, we describe the (preliminary) induced subgraphs that we use to cover $G$.

We will define one subgraph $G_{z}$ for each $z \in V$. Let $V_{z}$ (the vertex set of $G_{z}$ ) be

$$
V_{z}=\left\{x \in V:\left|\|x-z\|_{2}^{2}-\mu / 4\right| \leq 3 n / 4\right\} .
$$

The subgraph $G_{z}$ is the induced graph on $V_{z}$. First, we prove that these subgraphs $G_{z}$ do indeed cover the edges of $G$ : 
Lemma 2.2. Let $n \geq 2 C$. For all $(x, y) \in E$, there is a $z$ such that $x, y \in V_{z}$.

Proof. First we establish a simple claim that will help us choose an appropriate $z$ :

Claim 2.3. Let a be a vector in which the absolute value of each entry is at most $C$. Then there is a vector $w$ where each entry is $\pm 1 / 2$ such that $|(a, w)|=\left|\sum_{i=1}^{n} a_{i} w_{i}\right| \leq C / 2$ $\leq n / 4$.

Proof. We can prove this by induction by considering the partial sum $\sum_{i=1}^{r} a_{i} w_{i}$ which we will assume is at most $C / 2$ in absolute value. We can choose $w_{r+1}$ so that $a_{r+1} w_{r+1}$ has the opposite sign of this partial sum and this implies that the partial sum $\sum_{i=1}^{r+1} a_{i} w_{i}$ is also at most $C / 2$ in absolute value (although the sign may have changed). This completes the proof of the claim.

Let $a$ be a vector defined as follows: if $y_{i}-x_{i}$ is even, set $a_{i}=0$, and otherwise set $a_{i}=y_{i}-x_{i}$. We can apply Claim 2.3 to $a$, but furthermore change the values of $w$ to be zero on indices on which $a$ is zero. Then $|(a, w)|$ is still at most $C / 2$, and $w_{i}$ is $\pm 1 / 2$ whenever $a_{i}$ is non-zero, and zero whenever $a$ is zero. Set $z=(y+x) / 2+w$. Note that $z \in V$ because whenever $\left(y_{i}+x_{i}\right) / 2$ is not an integer, $a_{i}$ must be non-zero and hence $w_{i}$ is $\pm 1 / 2$ and alternatively whenever $\left(y_{i}+x_{i}\right) / 2$ is an integer, $a_{i}$ is zero and hence $w_{i}$ is zero. Consider the quantity

$$
\|z-x\|_{2}^{2}=\left\|\frac{y-x}{2}+w\right\|_{2}^{2}=\frac{1}{4}\|y-x\|_{2}^{2}+(y-x, w)+\|w\|_{2}^{2} .
$$

Since $x$ and $y$ are adjacent in $G$, we have $\left|\frac{1}{4}\|y-x\|_{2}^{2}-\mu / 4\right| \leq n / 4$. Also, $\|w\|_{2}^{2} \leq n / 4$. Finally, $(y-x, w)=(a, w)$ since $w$ is zero iff $a$ is zero. Hence $\left|\|z-x\|_{2}^{2}-\mu / 4\right| \leq$ $n / 4+C / 2+n / 4 \leq 3 n / 4$, and an identical argument holds for bounding $\left|\|z-y\|_{2}^{2}-\mu / 4\right|$. Thus $x, y \in V_{z}$ and the edge $(x, y)$ is covered by some induced subgraph $G_{z}$.

Next we establish that the maximum degree of any induced subgraph $G_{z}$ is not too large:

Lemma 2.4. For all $z \in V$, the maximum degree of $G_{z}$ is at most $(10.5)^{n}$.

Proof. Let $x \in V_{z}$ and consider any neighbor $y$ of $x$ that satisfies $y \in V_{z}$. We first establish that $x$ and $y$ are close to being antipodal in the ball centered at $z$, and hence we can bound the number of neighbors of $x$ in $V_{z}$ by bounding the number of points of $V$ in some small spherical cap around the antipodal point to $x$.

Define $x^{\prime}=2 z-x$, the antipodal point to $x$ with respect to the ball centered at $z$.

Consider the parallelogram $(x, z, y, x+y-z)$. By the parallelogram law, the sum of the squares of the four side lengths equals the sum of the squares of the lengths of the two diagonals. Therefore we obtain

$$
\|x-y\|_{2}^{2}+\|x+y-2 z\|_{2}^{2}=2\|x-z\|_{2}^{2}+2\|y-z\|_{2}^{2} .
$$

Using the definition of $x^{\prime}$, this gives

$$
\|x-y\|_{2}^{2}+\left\|y-x^{\prime}\right\|_{2}^{2}=2\|x-z\|_{2}^{2}+2\|y-z\|_{2}^{2} .
$$


Hence $\left\|y-x^{\prime}\right\|_{2}^{2}=2\|x-z\|_{2}^{2}+2\|y-z\|_{2}^{2}-\|x-y\|_{2}^{2}$ and as both $\|x-z\|_{2}^{2}$ and $\|y-z\|_{2}^{2}$ are approximately $\mu / 4$ (since $x, y \in V_{z}$ ) and $\|x-y\|_{2}^{2}$ is approximately $\mu$ because $x$ is adjacent to $y$ in $G$, this implies that $\left\|y-x^{\prime}\right\|_{2}^{2} \leq 4 n$. Therefore we can bound the degree of $x$ in $G_{z}$ by the number of lattice points in a ball of radius $2 \sqrt{n}$ (centered at the lattice point $x^{\prime}$ ). The unit $n$-dimensional cubes centered at these lattice points are pairwise disjoint, each has volume 1 , and they are all contained in a ball of radius $2 \sqrt{n}+0.5 \sqrt{n}=2.5 \sqrt{n}$. Therefore, the number of these points does not exceed the volume of an $n$-dimensional ball of radius $2.5 \sqrt{n}$. Since $n$ is even, the volume of this ball is

$$
\frac{\pi^{n / 2}(2.5 \sqrt{n})^{n}}{(n / 2) !}<(2 \pi e)^{n / 2} \frac{(2.5 \sqrt{n})^{n}}{n^{n / 2}}=(2.5 \cdot \sqrt{2 \pi e})^{n}<10.5^{n},
$$

where we have used the fact that $b !>(b / e)^{b}$ for any positive integer $b$. This completes the proof of the lemma.

Lemma 2.5. Let $H$ be a graph with maximum degree $d$. Then $H$ can be covered by $O\left(d^{2}\right)$ induced matchings.

Proof. Call two edges $e_{1}, e_{2}$ of $H$ in conflict if either they share a common end or there is an edge in $H$ connecting an endpoint of $e_{1}$ to an endpoint of $e_{2}$. It is clear that any edge $e$ of $H$ can be in conflict with at most $2 d-2+(2 d-2)(d-1)<2 d^{2}$ other edges of $H$.

Thus we can initialize each member of a set of $2 d^{2}$ induced matchings $M_{i}$ to be the empty set, and for each edge $e$ of $H$ in its turn, add $e$ to the first $M_{i}$ for which $e$ is not in conflict with any edge currently in $M_{i}$. Since $e$ is in conflict with less than $2 d^{2}$ edges, it can be added to some $M_{i}$. We can continue this procedure, obtaining a set of less than $2 d^{2}$ induced matchings covering all edges of $H$.

It follows that we can decompose the edges of each induced subgraph $G_{z}$ into at most $O\left(d^{2}\right) \leq O\left((10.5)^{2 n}\right)$ induced matchings, and this yields a decomposition of $G$ into $O\left(N d^{2}\right)$ induced matchings. These matchings can additionally be made edge-disjoint, since, if any edge is multiply covered we can remove it from all but one of the induced matchings (and the result is still an induced matching). We have thus proved the following.

Theorem 2.6. For every $n, C$ with $n \geq 2 C$, $n$ even, there is a graph $G$ on $N=C^{n}$ vertices that misses at most $N^{g}$ edges for

$$
g=2-\frac{1}{2 C^{4} \log C}+o(1)
$$

and can be covered by $N^{f}$ disjoint induced matchings, where

$$
f=1+\frac{2 \log 10.5}{\log C}+o(1) .
$$

Hence, for any $\epsilon>0$ we can construct a graph $G$ on $N$ vertices missing at most $N^{2-\delta}$ edges for $\delta=\delta(\epsilon)=e^{-O(1 / \epsilon)}$, that can be covered by $N^{1+\epsilon}$ pairwise disjoint induced matchings. Note that the number of matchings is nearly linear. Note also that by splitting 
each of these matchings $M$ into $\lfloor|M| / r\rfloor$ pairwise disjoint matchings, each of size exactly $r=N^{1-\epsilon-\delta}$, omitting the remaining $|M|-r\lfloor|M| / r\rfloor<r$ edges, we get an $(r, t)$-RS graph, where $r=N^{1-\epsilon-\delta}$ and the number of missing edges is at most $2 N^{2-\delta}$. As $\epsilon$ (and hence $\delta<\epsilon$ ) can be chosen to be arbitrarily small, this gives, with the right choice of parameters, an $(r, t)$-RS graph on $N$ vertices, with $r=N^{1-o(1)}$ and $r t=\left(\begin{array}{c}N \\ 2\end{array}\right)-o\left(N^{2}\right)$.

\subsection{A construction using error correcting codes}

Here we construct nearly complete graphs with large induced matchings using error correcting codes. These constructions will be incomparable to those in the previous sectionthe number of missing edges will be much smaller (in fact, the number of missing edges can be made asymptotically optimal as we will demonstrate in Section 3.2), but the price we pay is that the average size of an induced matching will only be a small power of $N$ as opposed to nearly linear. As we will show, the construction in this section will be better tailored to the application in [8] (at least for some values of the relevant parameters) than the construction of the previous section.

Throughout this section, we will use codes over the binary alphabet as well as over a bigger alphabet. Let $d_{\mathrm{H}}(x, y)$ be the Hamming distance between two binary strings $x$ and $y$ (of the same length). The Hamming weight of a binary string $x$ is the number of non-zero entries, or equivalently the Hamming distance to the all-zeros vector. We can similarly define the Hamming distance $d_{\mathrm{H}}(x, y)$ between two vectors $x$ and $y$ over a larger alphabet $[C]$ as the number of indices where these vectors disagree.

Definition 2.7. An $[n, k, d]$ linear code is a subspace $\mathcal{C}$ consisting of $2^{k}$ length $n$ binary vectors such that for all $x, y \in \mathcal{C}$ and $x \neq y, d_{\mathrm{H}}(x, y) \geq d$. We will call $n$ the encoding length, $k$ the dimension, and $d$ the distance of the code.

An $n \times k$ matrix $A$ of full column rank over GF(2) is the generating matrix of a code of dimension $k$ and length $n$ consisting of all linear combinations of its columns. The distance of this code is exactly the minimum Hamming weight of any non-zero code word. Throughout this section we will make use of a particular type of code:

Definition 2.8. Call a linear code $\mathcal{C}$ proper if the all-ones vector $\overrightarrow{1}$ is a codeword.

It is well-known that there are linear codes that achieve the Gilbert-Varshamov bound. In fact, proper codes also achieve this bound:

Lemma 2.9. If $\sum_{i=0}^{d}\left(\begin{array}{c}n \\ i\end{array}\right) \leq 2^{n-k}$, then there is a proper $[n, k, d]$ code. Thus, there is such a code in which $k=(1-H(d / n)) n$, where $H(x)=-x \log _{2} x-(1-x) \log _{2}(1-x)$ is the binary entropy function.

Proof. Let $\mathcal{C}$ be a maximum subspace of $\{0,1\}^{n}$ that contains $\overrightarrow{1}$ and has distance at least $d$. Such a subspace exists since $\{\overrightarrow{0}, \overrightarrow{1}\}$ has this property. Since $\mathcal{C}$ is a subspace, the minimum distance is exactly the minimum Hamming weight of any non-zero vector in $\mathcal{C}$. Let $w$ be some vector not in $\mathcal{C}$. If $d_{\mathrm{H}}(w, x) \geq d$ for every $x \in \mathcal{C}$, then it is easy to see that 
$(w+\mathcal{C}) \cup \mathcal{C}$ is a larger subspace containing $\overrightarrow{1}$ which still has distance at least $d$. Therefore, by the maximality of $\mathcal{C}$, we have

$$
\bigcup_{x \in \mathcal{C}}\left\{y: d_{\mathrm{H}}(y, x) \leq d\right\}=\{0,1\}^{n}
$$

and hence $|\mathcal{C}| \sum_{i=0}^{d}\left(\begin{array}{c}n \\ i\end{array}\right) \geq 2^{n}$. Since $\sum_{i=0}^{d}\left(\begin{array}{c}n \\ i\end{array}\right) \leq 2^{n-k}$, this implies that $|\mathcal{C}| \geq 2^{k}$.

Claim 2.10. Let $A$ be the generating matrix for a proper $[n, k, d]$ code $\mathcal{C}$ with $d>1$. Then deleting any row of $A$ results in a generating matrix $A^{\prime}$ for a proper $[n-1, k, d-1]$ code.

Proof. Note that $\mathrm{C}^{\prime}=\left\{A^{\prime} x: x \in\{0,1\}^{k}\right\}$ and hence $\mathrm{C}^{\prime}$ (defined by the generating matrix $A^{\prime}$ ) has dimension $k$, as no non-trivial linear combination of the columns of $A^{\prime}$ can be the zero vector, by the assumption $d>1$. The all-ones vector is still a codeword since $A x=\overrightarrow{1}$ implies that $A^{\prime} x=\overrightarrow{1}$. Finally, the minimum distance of $\mathcal{C}^{\prime}$ is the minimum Hamming weight of any non-zero codeword, and the Hamming weight of any codeword in $\mathcal{C}$ decreases by at most 1 by deleting any index.

Throughout this section let $\mathrm{C}=\mathcal{C}_{n}$ be an $[n, k, d]$ code, and let $\mathcal{C}_{n-1}, \mathcal{C}_{n-2}, \ldots, \mathcal{C}_{n-d+1}$ be proper $[n-1, k, d-1],[n-2, k, d-2], \ldots$ and $[n-d+1, k, 1]$ codes, respectively.

Next, we define a graph $G=(V, E)$ that will be the focus of this section. Let $V=$ $[C]^{n}$ and set $N=|V|=C^{n}$. Consider two vertices $a, b \in V$, where $a=\left(a_{1}, \ldots, a_{n}\right)$ and $b=\left(b_{1}, \ldots, b_{n}\right)$ for $a_{i}, b_{i} \in[C]$. There is an edge between $a$ and $b$ if and only if $d_{\mathrm{H}}(a, b)=\sum_{i=1}^{n} 1_{a_{i} \neq b_{i}}>n-d$.

It is easy to count the number of missing edges. Indeed, in the complement of $G$ each vertex $a$ is connected to all vertices $b$ so that $a_{i}=b_{i}$ for at least $d$ indices $i$. As the number of missing edges is half the sum of degrees in the complement, this gives:

Claim 2.11.

$$
\left(\begin{array}{l}
N \\
2
\end{array}\right)-|E| \leq \frac{1}{2} C^{n} \sum_{i=d}^{n}\left(\begin{array}{l}
n \\
i
\end{array}\right)(C-1)^{n-i} .
$$

Lemma 2.12. If $d / n \geq 2 /(C-1)$ then

$$
\frac{1}{2} C^{n} \sum_{i=d}^{n}\left(\begin{array}{l}
n \\
i
\end{array}\right)(C-1)^{n-i} \leq\left(\begin{array}{l}
n \\
d
\end{array}\right) C^{n}(C-1)^{n-d} .
$$

Proof. Using the inequality $\left(\begin{array}{l}n \\ i\end{array}\right) \leq(n / d)^{i-d}\left(\begin{array}{l}n \\ d\end{array}\right)$ we obtain a bound

$$
\sum_{i=d}^{n}\left(\begin{array}{l}
n \\
i
\end{array}\right)(C-1)^{n-i} \leq\left(\begin{array}{l}
n \\
d
\end{array}\right)(C-1)^{n-d} \sum_{j=0}^{n-d}(n / d)^{j}(C-1)^{-j}
$$

which implies the lemma.

Hence the number of missing edges in $G$ is at most $N^{e}$ for

$$
e=1+\frac{H(d / n)+(1-d / n) \log _{2}(C-1)}{\log _{2} C}+o(1) .
$$


Next, we describe the induced matchings that are used to cover the edges in $G$. In order to do so, we will define an equivalence relation over edges of $G$. In particular, this will be an equivalence relation over ordered pairs $(a, b)$, where $a=\left(a_{1}, \ldots, a_{n}\right)$ and $b=\left(b_{1}, \ldots, b_{n}\right)$, under the condition that $d_{\mathrm{H}}(a, b)>n-d$.

Definition 2.13. Let $S \subset[n],|S|=r$ and let $(a, b)$ be a pair of vertices in $V$ where $S=$ $\left\{i: a_{i}=b_{i}\right\}$. Let $x$ be a $\{0,1\}^{n-r}$ vector. Let $[n]-S=\left\{i_{1}, \ldots, i_{n-r}\right\}$ and $i_{1}<\cdots<i_{n-r}$. Then the $x$-flip of $(a, b)$ is a pair $(c, d)$ such that for all $i \in S, c_{i}=a_{i}=b_{i}=d_{i}$ and for all $i=i_{j} \notin S$ (i.e. $i$ is the $j^{\text {th }}$ smallest index not in $S$ ), $c_{i}=a_{i}, d_{i}=b_{i}$ if $x_{j}=0$ and otherwise $c_{i}=b_{i}, d_{i}=a_{i}$.

Informally, the $n-r$ indices not in $S$ are mapped in order to the $n-r$ bits in $x$ and the corresponding locations in $a$ and $b$ are swapped if and only if the corresponding bit of $x$ is 1 .

Definition 2.14. We will define a pair $(a, b) \sim\left(a^{\prime}, b^{\prime}\right)$ iff $S=\left\{i: a_{i}=b_{i}\right\}=S^{\prime}=$ $\left\{i: a_{i}^{\prime}=b_{i}^{\prime}\right\},|S|=r<d$ and furthermore there is an $x \in \mathcal{C}_{n-r}$ such that $\left(a^{\prime}, b^{\prime}\right)$ is the $x$-flip of $(a, b)$.

Next we will establish that this relation $\sim$ is indeed an equivalence relation, and that it is actually a relation on unordered pairs, that is, $(a, b) \sim(b, a)$ for all $a, b$ :

Claim 2.15. $(a, b) \sim(b, a)$.

This follows because the code $\mathcal{C}_{n-r}$ is proper (for all $r<d$ ), and hence the all-ones vector $\overrightarrow{1}$ lies in $\mathcal{C}_{n-r}$ and $(b, a)$ is the $\overrightarrow{1}$-flip of $(a, b)$.

Claim 2.16. $(a, b) \sim(c, d)$ iff $(c, d) \sim(a, b)$.

Proof. By symmetry we only need to establish one direction. Suppose $(a, b) \sim(c, d)$. Then $S=\left\{i: a_{i}=b_{i}\right\}=S^{\prime}=\left\{i: c_{i}=d_{i}\right\}$. Let $(c, d)$ be an $x$-flip of $(a, b)$ (where $\left.x \in \mathcal{C}_{n-r}\right)$. Then $(a, b)$ is also the $x$-flip of $(c, d)$.

Claim 2.17. $(a, b) \sim(c, d)$ and $(c, d) \sim(e, f)$ implies $(a, b) \sim(e, f)$.

Proof. Again note that $S=\left\{i: a_{i}=b_{i}\right\}=S^{\prime}=\left\{i: c_{i}=d_{i}\right\}=S^{\prime \prime}=\left\{i: e_{i}=f_{i}\right\}$. Let $x, y \in \mathcal{C}_{n-r}$ be such that $(c, d)$ is the $x$-flip of $(a, b)$ and $(e, f)$ is the $y$-flip of $(c, d)$. Then $x+y \in \mathcal{C}_{n-r}$ since the code is linear, and $(e, f)$ is the $x+y$-flip of $(a, b)$.

This immediately implies:

Lemma 2.18. The relation $\sim$ is an equivalence relation over unordered pairs $(a, b)$ which have Hamming distance $>n-d$.

Since each code $\mathcal{C}_{n-r}$ (for $r<d$ ) has dimension $k$, each equivalence class has size exactly $2^{k}$.

Lemma 2.19. Each equivalence class is an induced matching consisting of $2^{k-1}$ edges. 
Proof. Consider two edges $(a, b)$ and $(e, f)$ in the same equivalence class. Let $S=\{i$ : $\left.a_{i}=b_{i}\right\}=\left\{i: e_{i}=f_{i}\right\}$ where $|S|=r(<d)$. Let $(e, f)$ be the $x$-flip of $(a, b)$ for $x \in \mathcal{C}_{n-r}$. Since the code $\mathcal{C}_{n-r}$ has distance at least $d-r$, the Hamming weight of $x$ is at least $d-r$. Consider the Hamming distance between $a$ and $f$. Each index $i \in S$ is an index at which $a$ and $f$ agree (i.e. $a_{i}=f_{i}$ ). Furthermore, there is a bijection between indices in $x$ that are set to one and indices outside of the set $S$, for which $a$ and $f$ agree. So the vectors $a$ and $f$ agree on at least $r+(d-r)=d$ indices, and hence $a f$ is not an edge in $G$. Since $(e, f)$ and $(f, e)$ are in the same equivalence class, the above argument also shows that $a e, b e$ and $b f$ are non-edges.

If we use one induced matching for each equivalence class, then each edge in $G$ is covered exactly once and hence the number of induced matchings needed to cover $G$ is $|E| / 2^{k-1} \leq N^{2} / 2^{k}$

Theorem 2.20. For every $n, d, C$ such that $1 / 2>d / n \geq 2 /(C-1)$, there is a graph $G$ on $N=C^{n}$ vertices that misses at most $N^{e}$ edges for

$$
e=1+\frac{H(d / n)+(1-d / n) \log _{2}(C-1)}{\log _{2} C}+o(1)
$$

and can be covered by $N^{f}$ disjoint induced matchings, where

$$
f=2-\frac{1-H(d / n)}{\log _{2} C}+o(1) .
$$

In particular, for any $\epsilon>0$, there is a graph $G$ on $N$ vertices missing at most $N^{3 / 2+\epsilon}$ edges that can be covered by $N^{2-c \epsilon^{3}}$ induced matchings. This is obtained by choosing $C$ for which $\log _{2} C=\Theta(1 / \epsilon)$ and $d / n=1 / 2-\Theta(\epsilon)$.

Also we can choose $C=35$ and $d=0.15 n$, in which case $2(e-1), f<1.924$. Thus we can cover the edges of a complete graph on $2 N$ vertices by two graphs $G_{1}$ (set to $G$ with $N$ replaced by $2 N$ in the above construction) and $G_{2}$ (set to the complement of $G$ ), where the number of induced matchings needed to cover the edges of $G_{1}$ is $O\left(N^{2-\delta}\right)$ for $\delta>0.076$, and the same holds for $G_{2}$ (see Section 4.1 for details). For applications we need that the above statement holds also for covering all edges of the complete bipartite graph $K_{N, N}$ by two such graphs $G_{1}^{\prime}$ and $G_{2}^{\prime}$ - this clearly follows by splitting the vertices of $K_{2 N}$ arbitrarily into two equal classes and by defining $G_{i}^{\prime}$, for $i=1,2$, to be the graph obtained from $G_{i}$ by keeping only the edges that have one endpoint in each class.

\section{Limits}

\subsection{Triangle removal lemma}

The connection between the triangle removal lemma and the existence of $(r, t)$-RS graphs is well known since the work of Ruzsa and Szemerédi; for completeness we include the argument. 
Proposition 3.1. If there exists an $(r, t)$-RS graph on $N$ vertices, then there exists a graph on $N+t$ vertices with at least $3 r t / 2$ edges, in which every edge is contained in exactly one triangle. Thus one has to delete at least $r t / 2$ edges to destroy all triangles and yet the graph contains only $r t / 2$ triangles.

Proof. Let $G$ be an $(r, t)$-RS graph on $N$ vertices. Then its number of edges is $r t$ and hence, by a well known simple result, it contains a bipartite subgraph $G^{\prime}=\left(U, V, E^{\prime}\right)$ with at least $r t / 2$ edges. Clearly, these edges can be covered by $t$ induced matchings $M_{1}, \ldots, M_{t}$, and we can assume that these matchings are pairwise edge disjoint.

For each matching $M_{i}$, add an additional vertex $w_{i}$ and connect $w_{i}$ to the endpoints of all edges in $M_{i}$. The resulting graph $H=\left(U, V, W, E_{H}\right)$ is tripartite, has $N+t$ vertices and contains $\left|E^{\prime}\right|$ triangles. The critical property of this construction is that each edge of $H$ is in a unique triangle. Indeed, there is a natural set of $\left|E^{\prime}\right|$ triangles in $H$-each such triangle is specified by an edge $(u, v) \in E^{\prime}$ and if this edge is contained in the matching $M_{i}$, this edge is mapped to the triangle $\left(u, v, w_{i}\right)$ in $H$. There are in fact no other triangles in $H$ : Let $T=(a, b, c)$ be a triangle in $H$. Since $H$ is tripartite, there must be exactly one vertex from each set $U, V$ and $W$ in the set $a, b, c$. Suppose that $a \in U$ and $b \in V$. Then let $M_{i}$ be the unique matching containing the edge $(a, b)$. Suppose $c=w_{j} \neq w_{i}$. This implies that the matching $M_{j}$ covers both vertices $a$ and $b$ but does not contain the edge $(a, b)$, and hence $M_{j}$ is not an induced matching, a contradiction. This completes the proof.

The triangle removal lemma of [23], which is one of the early major applications of the regularity lemma, asserts that for any $\epsilon>0$ there is a $\delta=\delta(\epsilon)>0$ so that for $N>N(\epsilon)$ any graph on $N$ vertices from which one has to delete at least $\epsilon N^{2}$ edges to destroy all triangles contains at least $\delta N^{3}$ triangles. This and the above proposition imply that there are no $(r, t)$-RS graphs on $N$ vertices with $r=\Omega(N)$ and $t=\Omega(N)$. The original proof of [23] provides a rather poor quantitative relation between $\epsilon$ and $\delta$, but the improved recent proof of Fox [16] supplies better estimates (which are still very far from the known constructions). If the number of vertices is $N$ and the graph is a pairwise disjoint union of $t$ induced matching, each of size $r=c N$, then $t$ is at most $N / \log ^{(x)} N$ with $x=O(\log (1 / c))$, where $\log ^{(x)} N$ denotes the $x$-fold iterated logarithm. For more details, see [16].

\subsection{Reconstruction principle}

Here we prove lower bounds on the number of edges that a graph must miss if it can be covered by disjoint induced matchings of size $r$. These lower bounds establish that the results in Section 2.2 are essentially tight for an important range of the parameters. Indeed, as proved in that section, there are graphs on $N$ vertices missing $N^{3 / 2+\epsilon}$ edges that can be covered by disjoint, induced matchings of polynomial size. Yet, as we show below, any graph that can be covered by disjoint, induced matchings of size two or more must miss at least $N^{3 / 2}$ edges. We describe two proofs. The first is based on entropy considerations, and the second is an elementary combinatorial proof, which in fact yields a somewhat stronger result, as it bounds the minimum degree in the graph of missing 
edges. We believe, however, that both methods are interesting and each may have further applications. We start with the entropy proof.

Let $G=(V, E)$ be a graph on $N$ vertices that can be covered by disjoint induced matchings $M_{1}, \ldots, M_{t}$ each of size $r \geq 2$. We will prove an upper bound on the number $|E|$ of edges based on an application of the reconstruction principle (and through information-theoretic inequalities).

To this end, we define a random variable $A$ as follows:

- Choose $M_{i}$ uniformly at random.

- Choose an ordered set of two distinct edges $e_{1}, e_{2}$ from $M_{i}$.

Set $A=\left(e_{1}, e_{2}\right)$. Let $e_{1}=(W, X)$ and $e_{2}=(Y, Z)$. Here we use upper case letters to denote that each of these choices $W, X, Y$ and $Z$ is a random variable and we will use lower case letters to denote specific choices of these random variables.

Claim 3.2. $H(A)=\log |E|+\log (r-1)$.

Proof. Since we choose each matching $M_{i}$ uniformly at random, and each matching is of the same size $(r)$, the first edge $e_{1}$ is chosen uniformly at random from the set $E$. Conditioned on the choice of $e_{1}$, the remaining edge $e_{2}$ is chosen uniformly at random from the other $r-1$ edges in $M_{i}$.

Let $d_{v}$ be the number of missing edges incident to $v \in V$. Let $D_{v}$ be the set of nonneighbors of $v$, and let $f_{v}: D_{v} \rightarrow\left[d_{v}\right]$ be a function mapping each non-neighbor of $v$ to a unique integer in the set $\left[d_{v}\right]$.

- Choose $A$ as above and let $e_{1}=(w, x)$ and $e_{2}=(y, z)$.

- Choose $S_{1}$ with probability $1 / 2$ to be either $w$ or $x$, and let $S_{3}$ be the opposite choice.

- Choose $S_{2}$ with probability $1 / 2$ to be either $y$ or $z$.

We set the random variable $B=\left[s_{1}, f_{s_{1}}\left(s_{2}\right), f_{s_{2}}\left(s_{3}\right)\right]$.

Lemma 3.3. $H(B) \geq H(A)$.

Proof. We prove that $A$ can be computed as a deterministic function of $B$, and then we apply the chain rule for entropy to prove the lemma.

Claim 3.4. $A$ can be computed as a deterministic function of $B$.

Proof. Given $B$, we can compute $s_{2}$ using $s_{1}$ and $f_{s_{1}}\left(s_{2}\right)$, and using $s_{2}$ and $f_{s_{2}}\left(s_{3}\right)$ we can compute $s_{3}$. This in turn defines the edge $e_{1}=\left(s_{1}, s_{3}\right)$ which uniquely determines $M_{i}$ since the set of matchings disjointly covers the edges in $G$. From $M_{i}$ and $s_{2}$, we can compute the remaining edge $e_{2}$ : this is the unique edge incident to $s_{2}$ in the matching $M_{i}$.

The chain rule for entropy yields the expansion $H(B, A)=H(B)+H(A \mid B)$, but $H(A \mid B)=0$ because $A$ is a deterministic function of $B$. We can alternatively expand $H(B, A)$ as $H(A)+H(B \mid A)$. Since $H(B \mid A) \geq 0$ we get $H(B)=H(B, A) \geq H(A)$, as desired.

Next, we give an upper bound for the entropy of $B$ (based on the number of missing edges), and this combined with the lemma above will imply a contradiction if the number of missing edges is too small. 
Definition 3.5. We will call a random variable $S$ on $V$ degree-uniform if $S$ chooses a random vertex proportional to the degree in $G$.

Claim 3.6. $S_{1}$ and $S_{2}$ are degree-uniform random variables.

Note that these two random variables are not independent!

Proof. We can choose the random variable $A$ by choosing an edge uniformly at random from $E$, setting this edge to be $e_{1}$ and choosing $e_{2}$ uniformly at random from the remaining edges in the matching $M_{i}$ that contains $e_{1}$. The distribution of $S_{1}$ in this sampling procedure (for $A$ ) is clearly degree-uniform.

To prove the remainder of the claim, we can slightly modify the sampling procedure for $A$. We could instead choose an edge uniformly at random from $E$ and set this edge to be $e_{2}$. Then choose an edge $e_{1}$ uniformly at random from the other edges in the matching $M_{i}$ that contains $e_{2}$. This is an equivalent sampling procedure for generating $A$, and from this procedure it is clear that $S_{2}$ is degree-uniform.

Let $\bar{d}$ be the average degree in the complement of $G$.

Lemma 3.7. $H(B) \leq \log N+2 \log \bar{d}$.

Proof. We can decompose the random variable $B$ into $B_{1}=s_{1}, B_{2}=f_{s_{1}}\left(s_{2}\right)$ and $B_{3}=$ $f_{s_{2}}\left(s_{3}\right)$. Again, using the chain rule for entropy we obtain

$$
H(B)=H\left(B_{1}\right)+H\left(B_{2} \mid B_{1}\right)+H\left(B_{3} \mid B_{2}, B_{1}\right) .
$$

Since $S_{2}$ is a deterministic function of the random variables $B_{2}$ and $B_{1}$, we get

$$
H\left(B_{3} \mid B_{2}, B_{1}\right)=H\left(B_{3} \mid B_{2}, B_{1}, S_{2}\right) \leq H\left(B_{3} \mid S_{2}\right) .
$$

We can upper bound $H\left(B_{1}\right)$ by $\log N$, and

$$
H\left(B_{2} \mid B_{1}\right)=\sum_{s_{1}} \operatorname{Pr}\left[S_{1}=s_{1}\right] H\left(B_{2} \mid S_{1}=s_{1}\right) \leq \sum_{s_{1}} \operatorname{Pr}\left[S_{1}=s_{1}\right] \log d_{s_{1}} .
$$

Using Claim 3.6, this is

$$
H\left(B_{2} \mid B_{1}\right)=\sum_{s_{1}} \frac{N-1-d_{s_{1}}}{2|E|} \log d_{s_{1}} \leq \sum_{s_{1}} \frac{N-1-\bar{d}}{2|E|} \log \bar{d}=\log \bar{d},
$$

where we have used Jensen's inequality and the concavity of the functions $\log x$ and $-x \log x$. An identical bound holds also for $H\left(B_{3} \mid S_{2}\right)$ again using Claim 3.6, and thus we get $H(B) \leq \log N+2 \log \bar{d}$.

We can apply Lemma 3.3 and the bounds in Lemma 3.7 and Claim 3.2 to obtain the following theorem:

Theorem 3.8. Let $G=(V, E)$ be a graph on $N$ vertices that can be covered by disjoint induced matchings of size $r \geq 2$. Then the number of missing edges satisfies

$$
\left(\begin{array}{l}
N \\
2
\end{array}\right)-|E| \geq\left(\frac{1}{2 \sqrt{2}}-o(1)\right) r^{1 / 2} N^{3 / 2} .
$$


For $r=2$ the following construction shows that this estimate is tight up to a constant factor. Let $G$ be a graph on $N$ vertices such that $V(G)=\{(x, y): 1 \leq x, y \leq \sqrt{N}\}$ and every vertex $(x, y)$ is adjacent to all vertices $\left(x^{\prime}, y^{\prime}\right)$ such that $x \neq x^{\prime}$ and $y \neq y^{\prime}$. Then the complement of $G$ has $(\sqrt{N}-1) N$ edges and $G$ can be covered by the disjoint induced matchings $M_{x, x^{\prime}, y, y^{\prime}}=\left\{(x, y) \sim\left(x^{\prime}, y^{\prime}\right),\left(x, y^{\prime}\right) \sim\left(x^{\prime}, y\right)\right\}$ of size two.

We can also apply a nearly identical argument to the proof of Theorem 3.8 in the case in which $G$ is a bipartite graph:

Theorem 3.9. Let $G=(U, V, E)$ be a bipartite graph that can be covered by disjoint induced matchings of size $r \geq 3$. Then the number of missing edges satisfies

$$
|U| \times|V|-|E| \geq \Omega\left(r^{2 / 3}|U|^{2 / 3}|V|^{2 / 3}\right) .
$$

To prove this result, we choose $A^{\prime}$ to be three distinct edges from the matching $M_{i}$, and we use a length three path through pairs in $U \times V$ that are not in $E$ to define the corresponding random variable $B^{\prime}$. Again, the proof uses information-theoretic inequalities and the fact that (if appropriately defined) $A^{\prime}$ can be reconstructed as a deterministic function of $B^{\prime}$. It is worth noting that for a bipartite graph with $|U|=|V|=N$ and induced matchings of size 2, the following simple construction misses only $N$ edges. Let $G$ be a bipartite graph with $U, V=[N]$ such that every $i \in U$ is adjacent to all $j \in V, j \neq i$. Then the complement of $G$ has $N$ edges and $G$ can be covered by the disjoint induced matchings $M_{i, j}=\{(i, j),(j, i)\}$ of size two.

We can also give a direct counting argument, which is somewhat stronger, as it yields a lower bound on the minimum degree in the graph of missing edges. This counting argument proceeds by estimating the size of an appropriately defined set in two ways.

Theorem 3.10. If $G=(V, E)$ is a graph on $N$ vertices that is the disjoint union of induced matchings of size $r$, then the minimum degree $d$ in the complement of $G$ satisfies

$$
\left(\begin{array}{l}
d \\
2
\end{array}\right) \geq(r-1)(N-1-d) .
$$

Proof. Let $G$ be an edge disjoint union of the induced matchings $M_{1}, \ldots, M_{t}$ each of size $r$, and let $d_{v}$ be the degree of $v$ in the complement of $G$. Set

$$
\mathcal{F}_{v}=\left\{e \in E: v \notin e, \exists M_{i} \text { such that } e \in M_{i} \text { and } v \text { is covered by } M_{i}\right\} .
$$

For each $v \in V, \mathcal{F}_{v}$ contains precisely $\left(N-1-d_{v}\right)(r-1)$ edges since $v$ belongs to exactly $N-1-d_{v}$ matchings and for each such matching there are exactly $r-1$ choices of an edge (in the matching) that is not incident to $v$.

Alternatively, each $\mathcal{F}_{v}$ contains at most $\left(\begin{array}{c}d_{v} \\ 2\end{array}\right)$ edges: If $e \in \mathcal{F}_{v}$ then $v$ cannot be a neighbor of each endpoint of $e$ because the matching is induced. As there are at most $\left(\begin{array}{c}d_{v} \\ 2\end{array}\right)$ choices of pairs of vertices that are not neighbors of $v$, we find that for every $v \in V$,

$$
(r-1)\left(N-1-d_{v}\right) \leq\left(\begin{array}{c}
d_{v} \\
2
\end{array}\right) .
$$

In particular, this holds for the vertex of minimum degree $d$, completing the proof.

The assertion of Theorem 3.9 can also be proved by a counting argument. We omit the details. 


\section{Applications}

\subsection{Shared communication channels}

We apply our results to significantly improve the application in [8] of communicating over a shared directional multi-channel. Roughly, when communicating over a shared channel we want the edges (corresponding to messages sent in some time step called a round) to form an induced matching. Otherwise, a receiver will hear messages sent from two different sources and the messages will appear garbled. Birk, Linial and Meshulam construct graphs with positive density that can be covered by roughly $N^{2} / 24 r$ induced matchings where $r=(\log N)^{\Omega\left(\log \log N /(\log \log \log N)^{2}\right)}$. The authors then use these graphs to design a communication protocol for $N$ stations over a shared directional multi-channel where the round complexity of this protocol is $O\left(N^{2} / r\right)$. This is a slightly better than poly-logarithmic improvement over the naive protocol for bus-based architectures.

We can use our constructions to achieve a round complexity of $O\left(N^{2-\delta}\right)$ over a shared directional multi-channel. This is the first such protocol that provides a polynomial improvement over the naive protocol. We accomplish this using just one transmitter and two receivers per station. This corresponds to a partition of the edges of a complete bipartite graph into two graphs each of which can be decomposed into a small number of induced matchings. If we allow $C=C(\epsilon)$ receivers per station, we can achieve a round complexity that is $O\left(N^{1+\epsilon}\right)$ for any $\epsilon>0$ (here $N$ is a trivial lower bound). Hence, while previous protocols required a nearly quadratic number of rounds with a constant number of receivers per station, our protocols require only a nearly linear number of rounds.

Motivated by the application to communication over a shared channel, Meshulam [22] conjectured that any graph on $N$ vertices with positive density could not be covered by $O\left(N^{2-\delta}\right)$ induced matchings. The constructions presented in Sections 2.1 and 2.2 disprove this conjecture in a strong sense.

First we explain the model considered in [8]. Roughly, the goal is to design a good communication protocol using a small number of shared communication channels. More precisely, suppose we have $N$ stations, and each wants to send a (distinct) message to all the other stations. We further assume that each message is (roughly) the same size. In this context, it is often prohibitively expensive to build a point-to-point communication channel from each station to every other one. Often, the proposed solution is to use some form of a shared communication channel. Indeed, the standard bus-based architecture connects all pairs of stations using a single connection in such a way that only one message can be sent on the channel per time step and hence a total of $N^{2}$ rounds are needed to send all messages.

There are other architectures that can be implemented cheaply in hardware and can accomplish this task in a smaller number of rounds. One such architecture is the shared directional multi-channel. The combinatorial abstraction is that we imagine the communication graph as a complete bipartite graph $K_{N, N}$ (with $N$ vertices on the left, representing the transmitters of the stations, and $N$ vertices on the right, representing the receivers). A directed multi-channel allows us to partition $K_{N, N}$ into $C$ graphs $G_{1}, \ldots, G_{C}$. These graphs correspond to allocating $c$ receivers to each station. For each graph $G_{i}$, in each round we can exchange all messages corresponding to the edges in some induced match- 
ing in $G_{i}$ in one time step. These matchings are required to be induced because otherwise messages would interfere in the underlying hardware.

Thus the problem of designing a communication protocol for this architecture that completes in a small number of rounds and does not use too many transmitters and receivers per station is exactly the problem of covering all the edges of a complete bipartite graph (using at most $C$ graphs) so that the number of induced matchings needed to cover the edges in each graph is small. The number $C$ represents the number of receivers that each station must be equipped with, assuming it has only one transmitter, and so our goal is not only to minimize the number of rounds, but also to do so for a small value of $C$.

- For $C=2$, we give a protocol that completes in $O\left(N^{2-\delta}\right)$ rounds for $\delta>0.076$

- For any $\epsilon>0$, we show that there is a $C=C(\epsilon)=2^{O(1 / \epsilon)}$ so that there is a communication protocol that completes in $O\left(N^{1+\epsilon}\right)$ rounds.

Let $K_{N, N}$ be the complete bipartite graph with $N$ vertices on the left and $N$ on the right.

Theorem 4.1. There is a partition of the edges of $K_{N, N}$ into two graphs $G_{1}$ and $G_{2}$ so that each of these graphs can be covered by at most $O\left(N^{2-\delta}\right)$ induced matchings, for $\delta>0.076$.

Proof. This follows immediately from the construction given at the very end of Section 2.2: we can choose $G_{1}^{\prime}$ and $G_{2}^{\prime}$ that cover all edges of $K_{N, N}$, where $G_{1}^{\prime}$ covers all edges of $K_{N, N}$ but at most $N^{2-\delta}$ and yet it is a union of at most $N^{2-\delta}$ induced matchings. The second graph $G_{2}^{\prime}$ consists of all these remaining edges. This graph is in fact $d$-regular by construction and has $N^{e}$ edges, with $2(e-1)<1.924$. Since $d=N^{e-1}$, we can invoke Lemma 2.5 and we can cover $G_{2}^{\prime}$ by $O\left(d^{2}\right)=O\left(N^{2(e-1)}\right)$ induced matchings. The total number of induced matchings in each graph is thus at most $O\left(N^{2-\delta}\right)$.

Theorem 4.2. For any $\epsilon>0$, there is a $C=C(\epsilon)=2^{O(1 / \epsilon)}$ so that the edges of $K_{N, N}$ can be partitioned into $G_{1}, \ldots, G_{C}$ and each of these graphs can be covered by at most $O\left(N^{1+\epsilon}\right)$ induced matchings.

Proof. To obtain this result, we can instead invoke the construction in Section 2.1 to obtain a bipartite graph $G$ (obtained by splitting the vertices of the graph constructed in that section into two equal parts and by keeping all edges that join vertices in the two parts). For each $i$, we can take $G_{i}$ to be a random shift of $G$-i.e. we construct $G_{i}$ by permuting the labels of the vertices on the right randomly. $G$ misses less than $N^{2-\delta}$ edges, for $\delta=2^{-O(1 / \epsilon)}$, and hence if we take $C=2 / \delta$ random shifts the expected number of edges that are not covered in any $G_{i}$ is less than one. Hence there is some choice of $G_{1}, \ldots, G_{C}$ that covers the edges in the complete bipartite graph and yet the edges in each $G_{i}$ can be covered by at most $O\left(N^{1+\epsilon}\right)$ induced matchings. We note that the above proof can be derandomized using the method of conditional expectations, that is, the graphs $G_{i}$ can be generated efficiently and deterministically.

Finally we mention a simple lower bound for the number of rounds needed, proved by Meshulam [22]. This shows that for any constant number of receivers a super-linear number of rounds is needed: 
Proposition 4.3 ([22]). For any partition of the edges of $K_{N, N}$ into $G_{1}, \ldots, G_{C}$, the total number of induced matchings needed to cover $G_{1}, \ldots, G_{C}$ is at least $b(C) N^{1+1 /\left(2^{C}-1\right)}$.

Proof. We apply induction on $C$, the result for $C=1$ is trivial. Consider the case $C=2$. Without loss of generality, let $G_{1}$ contain at least half of the edges from the complete bipartite graph and suppose that the minimum number of induced matchings needed to cover $G_{1}$ is $N^{r}$. Then there is an induced matching (in this set) that contains at least $\frac{1}{2} N^{2-r}$ edges and hence $G_{2}$ contains a complete bipartite graph where the number of vertices on the left and on the right is at least $\frac{1}{4} N^{2-r}$. Hence the number of induced matchings needed to cover $G_{2}$ is at least $\frac{1}{16} N^{4-2 r}$. Since the quantity $\max \left(N^{r}, N^{4-2 r}\right)$ is minimized for $r=4 / 3$ the total number of induced matchings needed to cover $G_{1}$ and $G_{2}$ is at least $\Omega\left(N^{4 / 3}\right)$.

We can iterate the above argument in the general case. Without loss of generality let $G_{1}$ contain at least $(1 / C) N^{2}$ edges and suppose the minimum number of induced matchings needed to cover $G_{1}$ is $N^{r}$. Then the union of $G_{2}, \ldots, G_{C}$ contains a complete bipartite graph where the number of vertices on the left and on the right is at least $\frac{1}{2 C} N^{2-r}$. We can assume by induction that the total number of induced matchings needed to cover $G_{2}, \ldots, G_{C}$ is at least some $b^{\prime}(C) N^{(2-r)\left(1+1 /\left(2^{C-1}-1\right)\right)}$. The quantity

$$
\max \left(r,(2-r) \cdot \frac{2^{C-1}}{2^{C-1}-1}\right)
$$

is minimized for $r=2^{C} /\left(2^{C}-1\right)$, and this completes the proof.

Hence any protocol requires at least $\Omega\left(\log \frac{1}{\epsilon}\right)$ receivers per station to reduce the number of rounds to $O\left(N^{1+\epsilon}\right)$. In contrast, the protocol in Theorem 4.2 uses $2^{O(1 / \epsilon)}$ receivers per station to complete this same task in $O\left(N^{1+\epsilon}\right)$ rounds.

\subsection{Linearity testing}

Here we observe that our graphs can be plugged in the analysis of Håstad and Wigderson [20] of the graph test of Samorodnitsky and Trevisan [24] to provide a (modest) strengthening. We obtain slightly better bounds on the soundness of this test, which may be of interest for a particular range of parameters.

The classical linearity test of Blum, Luby and Rubinfeld chooses a pair of points $x$ and $y$ uniformly at random from the domain of a function, and checks if $f(x)+f(y)=$ $f(x+y)$. The test accepts $f$ if and only if this condition is met, and indeed this test always accepts a linear function and if $f$ is not linear, the probability that this test accepts $f$ can be bounded by $1 / 2+d(f) / 2$, where $d(f)$ is the maximum correlation of $f$ with a linear function [9].

What if we want to reduce the probability that a function $f$ that is not linear passes this test? We could perform $\ell$ independent trials, in which case the probability that $f$ is accepted is bounded by $(1 / 2+d(f) / 2)^{\ell}$. However, such a test queries the function $f$ on $3 \ell$ locations. Motivated by the problem of designing a PCP with optimal amortized query 
complexity and the related problem for linearity testing, Samorodnitsky and Trevisan introduced a graph-based linearity test: Associate each vertex in an $N$-vertex complete graph with a randomly chosen element from the domain of $f$, and for each edge check if $f(x)+f(y)=f(x+y)$ where $x$ and $y$ are the values associated with the endpoints of the edge. This test accepts if and only if all of these conditions are met.

This test queries the function $f$ on $N+\left(\begin{array}{c}N \\ 2\end{array}\right)$ locations and the hope is that the soundness should behave approximately like $\left(\begin{array}{c}N \\ 2\end{array}\right)$ independent trials of the original linearity test [9]. Samorodnitsky and Trevisan [24] showed that the soundness of this test is bounded by

$$
(1 / 2)^{\left(\begin{array}{l}
N \\
2
\end{array}\right)}+d(f) \text {. }
$$

This analysis was subsequently simplified and improved by Håstad and Wigderson [20], using the known existence of graphs that have many edges but can be covered by large (disjoint) induced matchings. The intuition behind this connection is that an induced matching corresponds to independent trials of the original Blum-Luby-Rubinfeld linearity test (although the formal analysis somewhat masks this intuition). Håstad and Wigderson [20] proved:

Theorem 4.4. If $G=(V, E)$ is an $(r, t)-R S$ graph, then the graph-test for $G$ accepts a function $f$ with probability at most

$$
e^{-(1-2 c)^{2} r t / 2}+d(f)^{c r}
$$

for any $0<c<1 / 2$.

This theorem is stated in [20] for $c=1 / 4$, but the tradeoff quoted above is immediate from their proof. Håstad and Wigderson [20] used the construction of Ruzsa and Szemerédi [23] mentioned in the introduction, which shows that there are $(r, t)$-RS graphs on $N$ vertices with $r=N / e^{O(\sqrt{\log N})}$ and $t=N / 3$.

We can plug our constructions directly into this theorem (with $\epsilon=o(1)$ ) to obtain slightly better bounds, for some special values of $d(f)$. Our constructions are dense, and hence improve the first term in the bound, but the second term is slightly worse (although we still have $\left.r=N^{1-o(1)}\right)$. Note that as the complete graph on $N$ vertices contains every graph on $N$ vertices, these bounds, like the ones of [24] and [20], provide the following upper estimate.

Theorem 4.5. The probability that the complete graph linearity test on $N$ vertices accepts a function $f$ is at most

$$
2^{-(1 / 2-o(1)) N^{2}}+d(f)^{N^{1-o(1)}} .
$$

In general, the bounds obtained will be better than either of those in [24] or [20] for some values of $d(f)$.

\subsection{The directed Steiner tree problem}

In this short subsection we briefly note the connection between our constructions and a candidate randomized rounding algorithm for the directed Steiner tree problem that motivated Vempala [27] to ask about the existence of certain $(r, t)$-RS graphs. 
Giving a poly-logarithmic approximation algorithm for the directed Steiner tree problem is a famous open problem in approximation algorithms. A special case is the group Steiner tree problem (in an undirected graph), for which Garg, Konjevod and Ravi gave an elegant, poly-logarithmic approximation algorithm [19]. Charikar et al. [13] give an approximation algorithm for the directed Steiner tree problem whose approximation guarantee is $\tilde{O}\left(N^{\epsilon}\right)$ for any $\epsilon>0$, and this guarantee can be made poly-logarithmic at the cost of running in quasi-polynomial time.

Even our understanding of the naive linear programming relaxation is quite weak. Zosin and Khuller [28] give an $\Omega(\sqrt{k})$ integrality gap (where $k$ is the number of terminals), but this construction has exponentially many (in $k$ ) vertices. Hence we could still hope that the naive relaxation has at most a poly-logarithmic (in $N$ ) integrality gap.

Rajaraman and Vempala considered a stronger relaxation and a candidate rounding algorithm. In the case in which the support of the solution to the linear program is a tree, they proved that their rounding algorithm achieves a poly-logarithmic approximation ratio and this analysis is reminiscent of the rounding procedure for the group Steiner tree problem [19].

However, even when flow merges in one layer of a layered graph (i.e. when the fractional solution is not supported on a tree), attempting to analyze the behavior of the rounding algorithm led Vempala to a combinatorial conjecture:

Conjecture 1 ([27]). Let $G=(U, V, E)$ be an $N \times k$ complete bipartite graph and $N \geq k$. Let $\mathcal{P}$ be a partition of the edge set and for a part $p \in \mathcal{P}$, let $p_{i}$ denote the degree of vertex $i$ in $p$ (i.e. the number of edges of $p$ incident to $i$ ). Then

$$
\sum_{i \in U, j \in V} \min \left(1, \sum_{p \in \mathcal{P}} \frac{p_{i} p_{j}}{|p|}\right) \geq C \frac{N k}{\log N}
$$

Our constructions yield a negative answer to the above conjecture. In our negative example we have $N=k$. To obtain this result, we can instead invoke the construction in Section 2.2 to obtain a bipartite graph $H$ (obtained by duplicating the vertices of the graph constructed in that section). We can take $\mathcal{P}$ to be the induced matchings covering $H$ and additionally we add a part in the partition (consisting of a single edge) for each edge across the bipartition missing from $H$.

We can upper bound the right hand side as

$$
\sum_{i \in U, j \in V} \min \left(1, \sum_{p \in \mathcal{P}} \frac{p_{i} p_{j}}{|p|}\right) \leq \sum_{(i, j) \in H} \sum_{p \in \mathcal{P}} \frac{p_{i} p_{j}}{|p|}+\sum_{(i, j) \notin H} 1 .
$$

$H$ is an $(r, t)$-RS graph (and $r=\Omega\left(N^{2-f}\right)$ in our construction) and so for each part $p$ we have $\sum_{(i, j) \in H} p_{i} p_{j} /|p|=1$ because $p$ is an induced matching with respect to $H$. The number of parts in the partition (ignoring singletons, which are not in $H$ anyway) is at most $O\left(N^{f}\right)$ and so we can bound the contribution of the first term by $O\left(N^{f}\right)$. Also, the number of edges that $H$ misses (across the bipartition) is at most $O\left(N^{e}\right)$ and hence we can bound the above sum by $O\left(N^{e}+N^{f}\right)$ for $e$ and $f$ as in Theorem 2.20 (recall that $N=k$ ). Since we can have both $e$ and $f$ at most 1.924 , it follows that the conjecture is false. 


\section{Concluding remarks and open questions}

We have given two constructions of nearly complete graphs that can be decomposed into large pairwise edge disjoint induced matchings and described several applications of these graphs.

The main combinatorial open problem that remains is to determine or estimate more precisely the set of all pairs $(r, t)$ so that there are $(r, t)$-RS graphs on $N$ vertices. This is interesting for most values of the parameters, but is of special interest in some specific range. In particular, if for $r=N /(\log N)^{g}$ with $g>1$, one can show that $t=o(N)$, this will improve the best known upper bound for the maximum possible cardinality of a subset of $\{1, \ldots, N\}$ with no 3 -term arithmetic progressions-a problem that received a considerable amount of attention over the years (see [25] and its references).

The study of the combinatorial problem above seems to require a variety of techniques: the known constructions of [23], [15], [22] and the ones given here apply tools from additive number theory, coding theory, low degree representations of Boolean functions and geometry, while the proofs of non-existence rely on the regularity lemma and on combinatorial and entropy based techniques. All of these, however, still leave a wide gap between the upper and lower bounds for at least some of the range, and it will be interesting to find additional ideas that will help to study this problem.

In all the applications considered here there are still remaining open problems. The communication protocols over a shared directional multi-channel we suggest, while improving substantially the existing ones, are still not optimal, and the problem of deciding the best possible number of rounds for $N$ stations, even with two receivers per station, is still not settled, although our results show that it is $N^{2-\delta}$ for some $\delta$ between 0.076 and $2 / 3$. The best possible upper bound for the probability of acceptance of a function $f$ in the linearity graph test, using a complete graph of size $N$, is also not precisely determined as a function of $N$ and $d(f)$ (although here the gap between the upper bounds and the lower bounds is not large-see [20]). Finally, it will be interesting to decide if our graphs can be helpful in establishing new integrality gap results for the natural relaxation of the directed Steiner tree problem, rather than merely estimating the performance of specific rounding schemes.

Our constructions can be extended to uniform hypergraphs as well. An induced matching in a $k$-uniform hypergraph is a set $M$ of pairwise vertex-disjoint edges so that no other edge of the hypergraph is contained in the set of all vertices covered by the members of $M$. We can describe an explicit construction showing that for every $k$ there are $k$-uniform hypergraphs on $N$ vertices with at least $(1-o(1))\left(\begin{array}{c}N \\ k\end{array}\right)$ edges, so that all edges can be partitioned into $N^{k-1+o(1)}$ pairwise edge disjoint induced matchings. The $o(1)$ terms here tend to zero as $N$ tends to infinity.

This construction has an interesting application in the study of a problem in communication complexity studied by Liang and Vaidya [21] and by Brody [10]. In this problem there are $k+1$ players, each having an $n$-bit string. The players are allowed point-to-point communication, and must decide, deterministically, if their inputs are all equal. In a trivial protocol the first $k$ players transmit their bits to the last one, who checks for equality and determines the answer. Thus a total communication of $k n$ bits suffices. Somewhat surpris- 
ingly, it turns out that one can do better. It is shown in [21] that at least $(k+1) n / 2$ bits of communication are needed, and the authors also obtain a non-trivial upper bound (which is not tight). Brody [10] has used our graphs to show that for $k+1=3,1.5 n+o(n)$ bits suffice, showing that the lower bound is tight for $k+1=3$ up to a low order additive error term. (In fact, the original construction of Ruzsa and Szemerédi suffices here). Using our construction for $k$-uniform hypegraphs we can extend his result and design a protocol that shows that for every $k$ and $n$, the minimum possible number of bits in a communication protocol for the above problem with $k+1$ players, each having an $n$-bit string, is $(1+o(1))(k+1) n / 2$. Brody and Håstad [10] have independently found a similar protocol, using the $k$ cliques of the graphs in our construction here.

The hypergraph construction may well have additional applications, and we hope to study it further in a subsequent work.

Acknowledgments. We thank Roy Meshulam and Santosh Vempala for helpful comments.

Research of N. Alon was supported in part by an ERC Advanced grant, by a USA-Israeli BSF grant, by the Israeli I-core program and by NSF grant No. DMS-0835373.

Research of A. Moitra was supported in part by NSF grant No. DMS-0835373 and by an NSF Computing and Innovation Fellowship.

Research of B. Sudakov was supported in part by NSF grant DMS-1101185, by AFOSR MURI grant FA9550-10-1-0569 and by a USA-Israel BSF grant.

\section{References}

[1] Alon, N.: Testing subgraphs in large graphs. In: Proc. 42nd IEEE FOCS, 434-441, IEEE (2001); also: Random Structures and Algorithms 21, 359-370 (2002) Zbl 1027.68095 MR 1948732 MR 1945375

[2] Alon, N., Fox, J.: Testing perfection is hard. To appear

[3] Alon, N., Shapira, A.: Testing subgraphs in directed graphs. In: Proc. $35^{\text {th }}$ ACM STOC, ACM Press, 700-709 (2003); also: J. Comput. System Sci. 69, 354-382 (2004) Zbl 1084.68087 MR 2087940

[4] Alon, N., Shapira, A.: A characterization of easily testable induced subgraphs. In: Proc. Fifteenth Annual ACM-SIAM SODA, 935-944 (2004); also: Combin. Probab. Comput. 15, 791805 (2006) MR 2271826

[5] Alon, N., Shapira, A.: On an extremal hypergraph problem of Brown, Erdős and Sós. Combinatorica 26, 627-645 (2006) Zbl 1121.05079 MR 2285626

[6] Barrington, D. A. M., Beigel, R., Rudich, S.: Representing Boolean functions as polynomials modulo composite numbers. Comput. Complexity 4, 367-382 (1994) Zbl 0829.68057 MR 1313536

[7] Behrend, F. A.: On sets of integers which contain no three terms in arithmetic progression. Proc. Nat. Acad. Sci. USA 32, 331-332 (1946) Z Zbl 0060.10302 MR 0018694

[8] Birk, Y., Linial, N., Meshulam, R.: On the uniform-traffic capacity of single-hop interconnections employing shared directional multichannels. IEEE Trans. Information Theory 39, 186-191 (1993) Zbl 0825.94164

[9] Blum, M., Luby, M., Rubinfeld, R.: Self-testing/correcting with applications to numerical problems. J. Comput. System Sci. 47, 549-595 (1993) Zbl 0795.68131 MR 1248868

[10] Brody, J.: Private communication (2012) 
[11] Brown, W. G., Erdős, P., Sós, V. T.: Some extremal problems on $r$-graphs. In: New Directions in the Theory of Graphs (Ann Arbor, 1971), Academic Press, New York, 55-63 (1973) Zbl 0258.05132 MR 0351888

[12] Brown, W. G., Erdős, P., Sós, V. T.: On the existence of triangulated spheres in 3-graphs and related problems. Period. Math. Hungar. 3, 221-228 (1973) Zbl 0269.05111 MR 0323647

[13] Charikar, M., Chekuri, C., Cheung, T-Y., Dai, Z., Goel, A., Guha, S., Li, M.: Approximation algorithms for directed Steiner problems. J. Algorithms 33, 73-91 (1999) Zbl 0937.68155 MR 1712693

[14] Erdôs, P., Frankl, P., Rödl, V.: The asymptotic number of graphs not containing a fixed subgraph and a problem for hypergraphs having no exponent. Graphs Combin. 2, 113-121 (1986) Zbl 0593.05038 MR 0932119

[15] Fischer, E., Newman, I., Raskhodnikova, S., Rubinfeld, R., Samorodnitsky, A.: Monotonicity testing over general poset domains. In: Proc. 34th Annual ACM Symposium on Theory of Computing, ACM, New York, 474-483 (2002) Zbl 1192.68359 MR 2121173

[16] Fox, J.: A new proof of the graph removal lemma. Ann. of Math. 174, 561-579 (2011) Zbl 1231.05133 MR 2811609

[17] Fox, J., Loh, P.: On a problem of Erdős and Rothschild on edges in triangles. Combinatorica 32, 619-628 (2012) MR 3063153

[18] Frankl, P., Füredi, Z.: Colored packing of sets in combinatorial design theory. Ann. Discrete Math. 34, 165-178 (1987) Zbl 0675.05020 MR 0920642

[19] Garg, N., Konjevod, G., Ravi, R.: A polylogarithmic approximation algorithm for the group Steiner tree problem. J. Algorithms 37, 66-84 (2000) Zbl 0962.68136 MR 1783249

[20] Håstad, J., Wigderson, A.: Simple analysis of graph tests for linearity and PCP. Random Structures Algorithms 22, 139-160 (2003) Zbl 1064.68070 MR 1954608

[21] Liang, G., Vaidya, N.: Multiparty equality function computation in networks with point-topoint links. In: Structural Information and Communication Complexity, Lecture Notes in Comput. Sci. 6796, Springer, 258-269 (2011) MR 2862845

[22] Meshulam, R.: Private communication (2011)

[23] Ruzsa, I., Szemerédi, E.: Triple systems with no six points carrying three triangles. In: Combinatorics (Keszthely, 1976), Colloq. Math. Soc. János Bolyai 18, North-Holland, 939-945 (1978) Zbl 0393.05031 MR 0519318

[24] Samorodnitsky, A., Trevisan, L.: A PCP characterization of NP with optimal amortized query complexity. In: Proc. 32nd Annual ACM Symposium on Theory of Computing, ACM, New York, 191-199 (2000) MR 2114532

[25] Sanders, T.: On Roth's theorem on progressions. Ann. of Math. 174, 619-636 (2011) Zbl pre05960714 MR 2811612

[26] Szemerédi, E.: Regular partitions of graphs. In: Problèmes combinatoires et théorie des graphes (Orsay, 1976), J. C. Bermond et al. (eds.), CNRS, Paris, 399-401 (1978) Zbl 0413.05055 MR 0540024

[27] Vempala, S.: Private communication (2011)

[28] Zosin, L., Khuller, S.: On directed Steiner trees. In: Proc. 13th Annual ACM-SIAM Symposium on Discrete Algorithms (San Francisco, 2002), SIAM, 59-63 (2002) Zbl 1093.68629 